\title{
Urinary biomarkers in prostate cancer: to the miRnome and beyond
}

\author{
Christianne Hoey ${ }^{1,2,3}$, Renu Jeyapala ${ }^{4,5}$, Paul C. Boutros ${ }^{1,6,7,8,9,10}$, Bharati Bapat ${ }^{4,5,11,12}$, Stanley K. Liu ${ }^{1,2,3}$ \\ ${ }^{1}$ Department of Medical Biophysics, University of Toronto, Toronto, Canada; ${ }^{2}$ Department of Radiation Oncology, University of Toronto, Toronto, \\ Canada; ${ }^{3}$ Sunnybrook-Odette Cancer Centre, Toronto, Canada; ${ }^{4}$ Institute of Medical Science, University of Toronto, Toronto, Canada; ${ }^{5}$ Lunenfeld- \\ Tanenbaum Research Institute, Sinai Health System, Toronto, Canada; ${ }^{6}$ Department of Human Genetics, ${ }^{7}$ Department of Urology, ${ }^{8}$ Broad Stem \\ Cell Research Center, ${ }^{9}$ Institute for Precision Health, ${ }^{10}$ Jonsson Comprehensive Cancer Center, University of California, Los Angeles, CA, USA; \\ ${ }^{11}$ Department of Surgery and Surgical Oncology, Division of Urology, University Health Network, Toronto, Canada; ${ }^{12}$ Department of Laboratory \\ Medicine and Pathobiology, University of Toronto, Toronto, Canada \\ Correspondence to: Stanley K. Liu, PhD, MD, FRCPC. Sunnybrook Health Sciences Centre, 2075 Bayview Avenue, Toronto, ON M4N 3M5, Canada. \\ Email: stanley.liu@sunnybrook.ca; Bharati Bapat, PhD. Lunenfeld-Tannenbaum Research Institute, 60 Murray Street, Toronto, ON M5T 3L9, \\ Canada. Email: bapat@lunenfeld.ca; Paul C. Boutros, PhD, MBA. University of California, Los Angeles, 10833 Le Conte Avenue, Los Angeles, CA \\ 90024, USA. Email: pboutros@mednet.ucla.edu. \\ Provenance and Peer Review: This article is commissioned and reviewed by the Section Editor Xiao Li (Department of Urology, Jiangsu Cancer \\ Hospital, Jiangsu Institute of Cancer Research, Nanjing Medical University Affiliated Cancer Hospital, Nanjing, China). \\ Response to: Connor AE, Jordan AC, Perry AS. Holding a MIRror up to the robustness of the prostate cancer urinary transcriptome. Transl Androl \\ Urol 2019. doi: 10.21037/tau.2019.09.25
}

Submitted Nov 06, 2019. Accepted for publication Nov 18, 2019.

doi: $10.21037 /$ tau.2019.11.25

View this article at: http://dx.doi.org/10.21037/tau.2019.11.25

We thank the Translational Andrology and Urology (TAU) editors for providing us this opportunity to reply to the insightful commentary by Conner et al. on our article "Temporal stability and prognostic biomarker potential of the prostate cancer urine transcriptome" (1). Liquid biopsies are one path leading to the future of personalized medicine. One of their most attractive features (pluralize), amongst many, is their non-invasive nature. From a small sample of blood or urine as much information can be gleaned as from a tumor biopsy, while sparing patients the risks of significant side effects like pain, bleeding and infection (2). Liquid biopsies have often been conjectured to yield a more representative picture of the significant spatial heterogeneity within an individual's tumor, although the quantitative support for that statement has not yet been developed for any solid cancer type, even those as spatially heterogeneous as prostate cancers (3). We showed that urine miRNA abundances correlate well with those in tissue derived from tumor tissue from the same patients, demonstrating their ability to represent true tumor biology (1). These features of liquid biopsies have not been overlooked: the field has exploded with a plethora of academic and commercial studies developing and evaluating non-invasive fluid biomarkers for cancer diagnosis, prognosis and monitoring of relapse. But quantity is not quality. A high-quality biomarker must be stable over time for successful downstream clinical application. The urinary miRnome demonstrates temporal stability, with hundreds of miRNAs showing similar abundances in longitudinal urine samples collected over at least several years.

A clinical setting where non-invasive biomarkers is especially needed is in Active Surveillance of prostate cancer. For patients with low-risk disease, close patient follow-up with repeat biopsies is used to monitor disease progression, sparing patients the costs and morbidities of definitive local therapy. While this procedure is highly effective, the use of repeat biopsies carries the inherent risk of complications mentioned above. We addressed this clinical need by identifying and validating a urinary miRNA signature for aggressive prostate cancer that distinguished low-risk (Gleason 6) from high-risk (Gleason 27 ) prostate cancer with an AUC of 0.74 (95\% CI, 0.55-0.92). Our 
signature may provide a non-invasive approach to improve the detection of aggressive disease in the context of active surveillance.

The anatomical placement of the prostate is a doubleedged sword. By virtue of its location, the prostatic urethra is removed during radical prostatectomy, which can result in urinary incontinence and negatively impact quality of life. But for biomarker development, the prostate's location around the urethra is a key advantage for non-invasively collecting prostate-specific markers from the urine. It has been well established by Pellegrini et al. and others that a digital rectal exam (DRE) preceding urine collection enriches prostate-specific gene transcripts (4). This highlights the importance of post-DRE urine collection for tumor interrogation. Although Conner et al. question the requirement of a DRE, our data supports the idea that it is imperative to provide enrichment of prostate-specific markers that might otherwise be missed. As such, all urine samples were obtained post-DRE and performed by two oncologists, Drs. S. Liu and D. Vesprini. This mitigates concerns of whether DREs were performed by only one individual, although of course it may be the case that more sensitive molecular technologies might eventually obviate the need for physical disruption of the prostate through a DRE. Conner et al. also thoughtfully raise the question of whether having multiple physicians performing the DRE could alter the longitudinal robustness of the signature. Our miRNA signature was temporally stable within individuals, with different physicians performing the DRE, so we would not expect to see any differences from DRE performance. But as they suggest, additional evidence of this in large multi-institutional cohort of pathologically, physiologically, and ethnically diverse patients will be essential to firmly demonstrate that any inter-clinician variability in DRE procedures does not reduce signature performance below clinically-relevant accuracies.

Many promising liquid biopsies exist for prostate cancer management, both as circulating blood-based assays and urine-based tests. Beyond our urinary miRNA signature, other urine-based tests for prostate cancer risk stratification include PCA3 (5,6), ExoDx (7), TMPRSS2:ERG (8), and ProCUrE (9). PCA3 is a post-DRE test used to guide physicians on whether a patient requires a biopsy or not $(5,6)$. Similar to PCA3, the ExoDx Prostate IntelliScore test is used to identify patients who should undergo a prostate biopsy. ExoDx uses the mRNA in non-DRE urine exosomes to differentiate patients with benign or low-risk (Gleason 6) vs. high-risk (Gleason $\geq 7$ ) prostate cancer (7). TMPRSS2:ERG is a post-DRE urine mRNA assay used to identify clinically-significant prostate cancer (8). ProCUrE is a post-DRE urine test utilizing DNA methylation signatures of six genes for detection and prognosis of prostate cancer (9) with an AUC of 0.795. Heads-up comparison of these tests in a controlled cohort powered for a specific well-defined clinical question is urgently needed to assess their relative performance and complementarity. Indeed studies integrating these tests with existing radiologic, clinical, genetic and epidemiologic risk information to develop multi-modal nomograms or other risk-assessment tools are key.

Monitoring with minimally invasive methods such as urine-based tests can increase patient compliance with Active Surveillance monitoring, reduce sampling bias and morbidity associated with repeat biopsies. What all these liquid biopsy tests-except ExoDx-have in common is the use of post-DRE urine samples. This consensus highlights the importance of the DRE for urinary biomarkers and demonstrates the feasibility of implementing a post-DRE urine-based biomarker test, and the urgent clinical need. The rapid aging of global populations is rapidly increasing the number of prostate biopsy procedures performed annually, with concomitant financial costs and patient risks.

The landscape of non-invasive biomarkers for prostate cancer risk stratification is rapidly evolving as promising signatures are continually discovered. From epigenomics to proteomics, we are no longer confined to one level of the genomic hierarchy when searching for biomarkers. A multiomic approach, which entails integrating individual '-omics' into a combined signature, boasts improved accuracy compared to their individual counterparts (10). This will require large-scale collaboration between healthcare professionals and interdisciplinary scientists to successfully develop and implement liquid biopsies for improved cancer management and patient outcomes.

\section{Acknowledgments}

Funding: This work was funded by The Canadian Cancer Society, Relay for Life (Odette Cancer Centre), and Prostate Cancer Fight Foundation, Huronia Chapter of the Motorcycle Ride for Dad to SKL and DV. Movember Prostate Cancer Canada Translation Acceleration Grant (TAG) No. 2014-01 to BB, PB, SK, DV. Ontario Student Opportunity Trust Funds Awards to RJ, EOM, FZ. CCSG: This work was supported by the NIH/NCI under award number P30CA016042 and by an operating grant from 
the National Cancer Institute Early Detection Research Network (1U01CA214194-01).

\section{Footnote}

Conflicts of Interest: All authors have completed the ICMJE uniform disclosure form (available at http://dx.doi. org/10.21037/tau.2019.11.25). PCB has a patent or multiple patients on prostate cancer biomarkers issued. The other authors have no conflicts of interest to declare.

Ethical Statement: The authors are accountable for all aspects of the work in ensuring that questions related to the accuracy or integrity of any part of the work are appropriately investigated and resolved.

Open Access Statement: This is an Open Access article distributed in accordance with the Creative Commons Attribution-NonCommercial-NoDerivs 4.0 International License (CC BY-NC-ND 4.0), which permits the noncommercial replication and distribution of the article with the strict proviso that no changes or edits are made and the original work is properly cited (including links to both the formal publication through the relevant DOI and the license). See: https://creativecommons.org/licenses/by-ncnd/4.0/.

\section{References}

1. Jeon J, Olkhov-Mitsel E, Xie H, et al. Temporal stability and prognostic biomarker potential of the prostate cancer urine transcriptome. J Natl Cancer Inst 2020;112:247-55.

Cite this article as: Hoey C, Jeyapala R, Boutros PC, Bapat B, Liu SK. Urinary biomarkers in prostate cancer: to the miRnome and beyond. Transl Androl Urol 2020;9(2):843-845. doi: $10.21037 /$ tau.2019.11.25
2. Loeb S, Vellekoop A, Ahmed HU, et al. Systematic review of complications of prostate biopsy. Eur Urol 2013;64:876-92.

3. Boutros PC, Fraser M, Harding NJ, et al. Spatial genomic heterogeneity within localized, multifocal prostate cancer. Nat Genet 2015;47:736-45.

4. Pellegrini KL, Patil D, Douglas KJS, et al. Detection of prostate cancer-specific transcripts in extracellular vesicles isolated from post-DRE urine. Prostate 2017;77:990-9.

5. Hessels D, Klein Gunnewiek JM, van Oort I, et al. DD3(PCA3)-based molecular urine analysis for the diagnosis of prostate cancer. Eur Urol 2003;44:8-15; discussion 15-6.

6. Roobol MJ, Schröder FH, van Leeuwen P, et al. Performance of the prostate cancer antigen 3 (PCA3) gene and prostate-specific antigen in prescreened men: exploring the value of $\mathrm{PCA} 3$ for a first-line diagnostic test. Eur Urol 2010;58:475-81.

7. McKiernan J, Donovan MJ, O'Neill V, et al. A Novel Urine Exosome Gene Expression Assay to Predict Highgrade Prostate Cancer at Initial Biopsy. JAMA Oncol 2016;2:882-9.

8. Tomlins SA, Aubin SM, Siddiqui J, et al. Urine TMPRSS2:ERG fusion transcript stratifies prostate cancer risk in men with elevated serum PSA. Sci Transl Med 2011;3:94ra72.

9. Zhao F, Olkhov-Mitsel E, Kamdar S, et al. A urine-based DNA methylation assay, ProCUrE, to identify clinically significant prostate cancer. Clin Epigenetics 2018;10:147.

10. Sinha A, Huang V, Livingstone J, et al. The Proteogenomic Landscape of Curable Prostate Cancer. Cancer Cell 2019;35:414-27.e6. 